\title{
Predictive Value of Plasma NGAL:Hepcidin-25 for Major Adverse Kidney Events After Cardiac Surgery with Cardiopulmonary Bypass: A Pilot Study
}

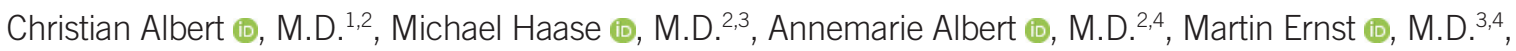

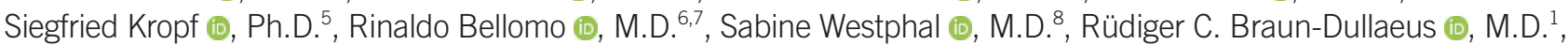 \\ Anja Haase-Fielitz (i), Pharm.D. ${ }^{9,10,11}$, and Saban Elitok (iD, M.D. ${ }^{4}$ \\ ${ }^{1}$ Medical Faculty, University Clinic for Cardiology and Angiology, Otto-von-Guericke University Magdeburg, Magdeburg, Germany; ${ }^{2}$ Diaverum Renal Services, \\ MVZ Potsdam, Potsdam, Germany; ${ }^{3}$ Medical Faculty, Otto-von-Guericke University Magdeburg, Magdeburg, Germany; ${ }^{4}$ Department of Nephrology and \\ Endocrinology, Klinikum Ernst von Bergmann, Potsdam, Germany; ${ }^{5}$ Institute for Biometrics and Medical Informatics, Otto-von-Guericke University \\ Magdeburg, Magdeburg, Germany; ${ }^{6}$ Department of Intensive Care, The Austin Hospital, Melbourne, Australia; ${ }^{7}$ Centre for Integrated Critical Care, \\ The University of Melbourne, Melbourne, Australia; ${ }^{8}$ nstitute of Laboratory Medicine, Hospital Dessau, Dessau, Germany; ${ }^{9}$ Department of Cardiology, \\ Immanuel Diakonie Bernau, Heart Center Brandenburg, Brandenburg Medical School Theodor Fontane, MHB, Germany; ${ }^{10}$ Institute of Social Medicine and \\ Health Systems Research, Otto-von-Guericke University Magdeburg, Magdeburg, Germany; ${ }^{11}$ Faculty of Health Sciences Brandenburg, Potsdam, Germany
}

Background: Neutrophil gelatinase-associated lipocalin (NGAL) and hepcidin-25 are involved in catalytic iron-related kidney injury after cardiac surgery with cardiopulmonary bypass. We explored the predictive value of plasma NGAL, plasma hepcidin-25, and the plasma NGAL:hepcidin-25 ratio for major adverse kidney events (MAKE) after cardiac surgery.

Methods: We compared the predictive value of plasma NGAL, hepcidin-25, and plasma NGAL:hepcidin-25 with that of serum creatinine $(\mathrm{Cr})$ and urinary output and protein for primary-endpoint MAKE (acute kidney injury [AKI] stages 2 and 3, persistent AKI >48 hours, acute dialysis, and in-hospital mortality) and secondary-endpoint AKI in 100 cardiac surgery patients at intensive care unit (ICU) admission. We performed ROC curve, logistic regression, and reclassification analyses.

Results: At ICU admission, plasma NGAL, plasma NGAL:hepcidin-25, plasma interleukin-6, and Cr predicted MAKE (area under the ROC curve [AUC]: 0.77, 0.79, 0.74, and 0.74 , respectively) and $\mathrm{AKI}(0.73,0.89,0.70$, and 0.69$)$. For AKI prediction, plasma NGAL:hepcidin-25 had a higher discriminatory power than $\mathrm{Cr}$ (AUC difference 0.26 [95\% $\mathrm{Cl} 0.00-0.53]$ ). Urinary output and protein, plasma lactate, C-reactive protein, creatine kinase myocardial band, and brain natriuretic peptide did not predict MAKE or AKI (AUC $<0.70)$. Only plasma NGAL:hepcidin-25 correctly reclassified patients according to their MAKE and AKI status (category-free net reclassification improvement: $0.82[95 \% \mathrm{Cl}$ 0.12-1.52], 1.03 [0.29-1.77]). After adjustment to the Cleveland risk score, plasma NGAL:hepcidin-25 $\geq 0.9$ independently predicted MAKE (adjusted odds ratio 16.34 [95\% Cl 1.77-150.49], $P=0.014$ ).

Conclusions: Plasma NGAL:hepcidin-25 is a promising marker for predicting postoperative MAKE.

Key Words: Acute kidney injury, Major adverse kidney events, Cardiac surgery, Cardiopulmonary bypass, Hepcidin, Neutrophil gelatinase-associated lipocalin, Serum creatinine
Received: October 5, 2020

Revision received: November 14, 2020

Accepted: January 13, 2021

\section{Corresponding author:}

Christian Albert, M.D.

Medical Faculty, University Clinic for Cardiology and Angiology, Otto-vonGuericke University, Leipziger Straße 44, 39120 Magdeburg, Germany and Diaverum Renal Services, Germany Diaverum MVZ Am Neuen Garten Am Neuen Garten 11, 14469 Potsdam,

Germany

Tel: +49-331-280690

Fax: +49-331-2806932

E-mail: Christian.Albert@Diaverum.com

\section{(i) (5)}

๔ Korean Society for Laboratory Medicine This is an Open Access article distributed under the terms of the Creative Commons Attribution Non-Commercial License (https://creativecommons.org/licenses/by-nc/4.0) which permits unrestricted non-commercial use, distribution, and reproduction in any medium, provided the original work is properly cited. 


\section{INTRODUCTION}

Acute kidney injury (AKI) frequently occurs after cardiac surgery and is independently associated with major adverse outcomes [1]. Routine postoperative risk assessment aims at earliest possible treatment adjustment [2]. However, the accuracy of clinical risk assessment for cardiac surgery-associated AKI is limited even when classical kidney function parameters, such as serum creatinine $(\mathrm{Cr})$ and urinary output and protein, or risk prediction systems such as the Cleveland risk score are used [3]. Thus, there is a need for new approaches for improved AKI risk prediction. Accordingly, in addition to classical kidney function parameters, kidney tubular injury biomarkers have been suggested to be included in kidney risk assessment [4].

Cardiopulmonary bypass causes inflammation and hemolysis with the generation of labile iron [5]. Inflammatory cytokines and labile iron appear to be important mediators of cardiac surgery-associated AKI [6, 7]. Neutrophil gelatinase-associated lipocalin (NGAL) and hepatic bactericidal protein (hepcidin-25) may be involved in the regulation of tubular iron metabolism [5, 7]. Plasma NGAL is involved in iron sequestration and is associated with tubular injury [8]. Postoperatively, decreased plasma concentrations of hepcidin-25 have been found in critically ill patients with severe AKI [6]. Levels of interleukin (IL)-6, a proinflammatory cytokine filtered by the glomeruli, were recently found to be elevated in the plasma and urine of patients with acute tubular injury [9]. Other biochemical markers, such as brain natriuretic peptide (BNP), lactate, creatine kinases, haptoglobin, hemoglobin, C-reactive protein (CRP), and leukocyte count may complementarily indicate postoperative systemic and circulatory conditions such as acute cardio-renal failure associated with fluid overload, hemolysis and hemorrhage, acute metabolic dysregulation, vasodilatation, and shock [10-12]. Biomarkers shed or directly released into the urine were thought to be superior in tubular injury detection; however, a recent metaanalysis showed advantages of plasma NGAL over urinary NGAL [13]. The predictive value of novel plasma biomarkers measured at admission to the intensive care unit (ICU) after cardiac surgery has never been compared with that of classical kidney function parameters and novel urinary parameters for major adverse kidney events (MAKE) or AKI. We aimed to identify the plasma kidney injury biomarker with the best predictive value for MAKE after cardiac surgery and to compare the predictive value of the identified biomarker with that of classical kidney function parameters and urinary biomarkers for postoperative kidney risk assessment.

\section{MATERIALS AND METHODS}

In reporting this prospective study, we followed the Strengthening the Reporting of Observational Studies in Epidemiology (STROBE) recommendations [14].

\section{Patients and setting}

This study used the control group of the Bicarbonate multicenter randomized controlled trial [15]. A previous study, assessing urinary biomarkers that improve the predictive power of a preoperative kidney injury risk score after cardiac surgery, also used biomarker measurement data for the control group of the above described randomized controlled trial [16]. One hundred patients with increased risk of AKI underwent elective cardiac surgery with cardiopulmonary bypass at the German Heart Center, Berlin, Germany (NCT00672334) between January 2009 and June 2010 [15]. The Institutional Review Board of Charité University of Berlin, Germany (approval no: ZS EK 11 654/07) approved this study. The ethics committee granted permission to collect data and conduct biomarker measurements. Written informed consent was obtained from each patient. Patients undergoing emergency surgery ( $<24$ hours between hospital admission and operation) or off-pump surgery, those presenting with advanced chronic kidney disease $(\mathrm{Cr}>300 \mu \mathrm{mol} / \mathrm{L})$ or requiring kidney transplant, those $<18$ years, those on immunosuppressive medication, and those enrolled in a conflicting study were excluded.

\section{Study endpoints}

The primary endpoint was the development of MAKE, defined as alternatively occurring events of Risk, Injury, Failure, Loss of kidney function, and End-stage kidney disease (RIFLE)-AKI stages of injury or failure; persistent AKI $>48$ hours; acute kidney replacement therapy (KRT) initiation; and in-hospital mortality. The secondary endpoint was AKI (stages 1-3) based on the RIFLE classification using Cr and urinary output criteria [17]. We chose the RIFLE criteria because of their better predictive value for in-hospital mortality in cardiac surgery patients than that of criteria based on the Acute Kidney Injury Network or Kidney Disease Improving Global Outcome Initiatives [18].

\section{Biomarker sampling and measurement}

Plasma was obtained at induction of anesthesia at ICU admission and at 24 hours postoperatively, as previously reported [15]. Plasma BNP $(\mathrm{ng} / \mathrm{L})$ and plasma NGAL $(\mathrm{ng} / \mathrm{mL})$ were measured using a point-of-care immunoassay (Triage NGAL-test, Triage 
BNP-test, Alere; Biosite Inc., San Diego, CA, USA). Plasma IL-6 $(\mathrm{pg} / \mathrm{mL})$ was determined using the Cobas e/c411 Immunoassay Analyzer Platform (Roche Diagnostics, Mannheim, Germany). Hepcidin-25 (ng/mL) was measured using a C-ELISA kit (Intrinsic Lifesciences, LLC, La Jolla, CA, USA), according to the manufacturer's instructions. Serum $\mathrm{Cr}$ was measured using a Cobas 8000 modular analyzer (Roche Diagnostics). Laboratory investigators were blinded to the sample sources and clinical outcomes.

\section{Statistical analysis}

We focused on the time of ICU admission because it is the time point at which maximum concentrations of postoperative plasma biomarker concentrations can be reasonably expected based on previous findings $[19,20]$ and because it is sufficiently early to allow effective intervention. The discriminative value and performance of candidate biomarkers for predicting study endpoints were assessed. In brief, the area under the ROC curve (AUC) (reported as AUC [95\% confidence interval
(CI)]) based on biomarker concentrations measured at ICU admission in conjunction with primary and secondary study endpoints was calculated separately for each marker (for hepcidin-25, haptoglobin, and hemoglobin with reversed orientation indicated, e.g., 1-hepcidin-25). Correlation coefficients were calculated using the Spearman's rank correlation test. The discriminative accuracy of a biomarker for predicting an event of interest was considered acceptable when univariate AUC $\geq 0.7$ $[21,22]$. AUCs were compared according to the method of Hanley and McNeil [23]. To assess the ability of a biomarker to independently predict the endpoints, we calculated the odds ratio (OR) $[\operatorname{Exp}(\mathrm{B})]$ adjusted to the following Cleveland kidney injury risk score variables: gender, congestive heart failure defined as New York Hearth Association classification class 3 or 4 or left ventricular ejection fraction $<35 \%$, chronic kidney disease defined as preoperative $\mathrm{Cr}>120 \mu \mathrm{mol} / \mathrm{L}$, insulin-dependent diabetes mellitus, chronic obstructive pulmonary disease, and history of cardiac surgery [3]. We evaluated improvements in the performance of the above-defined reference model following the

Table 1. Preoperative and intraoperative patient characteristics

\begin{tabular}{|c|c|c|c|c|c|c|}
\hline Variable & No MAKE (N=91) & MAKE $(\mathrm{N}=9)$ & $P^{*}$ & № AKI $(N=91)$ & AKI $(N=9)$ & $P^{\dagger}$ \\
\hline Age (yr) & & & 0.078 & & & 0.078 \\
\hline $25-50$ & $12(13.2)$ & $0(0)$ & & $12(13.2)$ & $0(0)$ & \\
\hline $51-70$ & $43(47.3)$ & $2(22.2)$ & & $43(47.3)$ & $2(22.2)$ & \\
\hline $71-90$ & $36(39.6)$ & $7(77.8)$ & & $36(39.6)$ & $7(77.8)$ & \\
\hline Preoperative eGFR <60 mL/min (CKD-EPI), N (\%) & $23(25.3)$ & $6(66.6)$ & 0.009 & $24(26.4)$ & $5(55.6)$ & 0.116 \\
\hline Left ventricular ejection fraction, \% & $50(40-60)$ & $55(25-60)$ & 0.609 & $50(40-60)$ & $55(25-60)$ & 0.739 \\
\hline Pulmonary hypertension, N (\%) & $26(28.6)$ & $3(3.3)$ & 0.716 & $26(28.6)$ & $3(3.3)$ & 0.716 \\
\hline Atrial fibrillation, $\mathrm{N}(\%)$ & $25(27.5)$ & $3(33.3)$ & 0.024 & $25(27.5)$ & $6(66.6)$ & 0.024 \\
\hline Peripheral vascular disease, N (\%) & $19(20.9)$ & $2(22.2)$ & $>0.99$ & $17(18.9)$ & $4(44.4)$ & 0.089 \\
\hline Diabetes mellitus on medication, $\mathrm{N}(\%)$ & $17(18.9)$ & $3(3.3)$ & 0.378 & $17(18.9)$ & $3(3.3)$ & 0.378 \\
\hline Hypercholesterolemia, N (\%) & $58(63.7)$ & $6(66.6)$ & $>0.99$ & $58(63.7)$ & $6(66.6)$ & $>0.99$ \\
\hline \multicolumn{7}{|l|}{ Preoperative medication } \\
\hline Renin-angiotensin-aldosterone system inhibitors, N (\%) & $47(51.7)$ & $7(77.8)$ & 0.134 & $46(50.5)$ & $8(88.9)$ & 0.036 \\
\hline$\beta$-blockers, N (\%) & $64(70.3)$ & $8(88.9)$ & 0.237 & $63(69.2)$ & $9(100)$ & 0.050 \\
\hline Calcium channel blockers, N (\%) & $30(33.0)$ & $4(44.4)$ & 0.485 & $30(33.0)$ & $4(44.4)$ & 0.485 \\
\hline Diuretics, N (\%) & $52(57.1)$ & $7(77.8)$ & 0.230 & $52(57.1)$ & $7(77.8)$ & 0.230 \\
\hline Statins, N (\%) & $48(52.8)$ & $5(55.6)$ & $>0.99$ & $48(52.8)$ & $5(55.6)$ & $>0.99$ \\
\hline Cross-clamp time, min & $72(56-89)$ & $96(65-153)$ & 0.055 & $73(56-89)$ & 66 (59-153) & 0.350 \\
\hline
\end{tabular}

Numbers denote median (25th-75th percentile) or $\mathrm{N}(\%)$ as appropriate.

${ }^{*} P$ for MAKE vs. no MAKE. ${ }^{\dagger} P$ for AKI vs. no AKI.

Abbreviations: AKI, acute kidney injury; CKD-EPI, Chronic Kidney Disease Epidemiology Collaboration equation for estimation of glomerular filtration rate; eGFR, estimated glomerular filtration rate; MAKE, major adverse kidney events. 
addition of a biomarker using category-free net reclassification improvement (cfNRI) and integrated discrimination improvement (IDI) [24]. We used leave-one-out cross-validation to quantify how accurately the reference and reclassification models would perform with independent data. We applied logarithmic transformations when necessary. Information regarding missing data is provided in tables or figure legends. We used SPSS, version 25.0 (IBM Corp., Armonk, NY, USA), MedCalc, version 19.5.2 (MedCalc Software Ltd., Ostend, Belgium), and SAS, version 9.4 (SAS Institute Inc., Cary, NC, USA) for statistical analysis. As this was an exploratory study, $P$-values are descriptive and were not adjusted for multiplicity.

\section{RESULTS}

\section{Patient enrollment}

Patient enrollment characteristics, including age distribution, comorbidities, preoperative medications, and cross-clamp time according to patient MAKE and AKI status are reported in Table
1. Patients who developed postoperative MAKE or AKI were more likely to be in the older age groups. The proportion of preoperative chronic kidney disease and atrial fibrillation was higher in patients with MAKE or AKI than in those without MAKE or AKI. Preoperative medication and cross-clamp time were similar in patients with and without MAKE or AKI (Table 1). Additional perioperative patient characteristics have been previously published [16]. Median plasma hepcidin-25 concentrations at ICU admission were higher in patients without MAKE (256 ng/mL [25th-75th percentile: 148-388] or AKI (260 ng/mL [25th-75th percentile: 152-384]) than in patients with subsequent MAKE (168 ng/mL [25th-75th percentile: 152-292] or AKI (168 ng/ $\mathrm{mL}$ [25th-75th percentile: 76-260]).

\section{Predictive performance of plasma biomarkers at ICU admission}

For the prediction of MAKE, plasma NGAL:hepcidin-25, plasma NGAL, plasma IL-6, and $\mathrm{Cr}$ had an AUC >0.7 (Fig. 1), while plasma NGAL:hepcidin-25, plasma NGAL, and $\mathrm{Cr}$ predicted

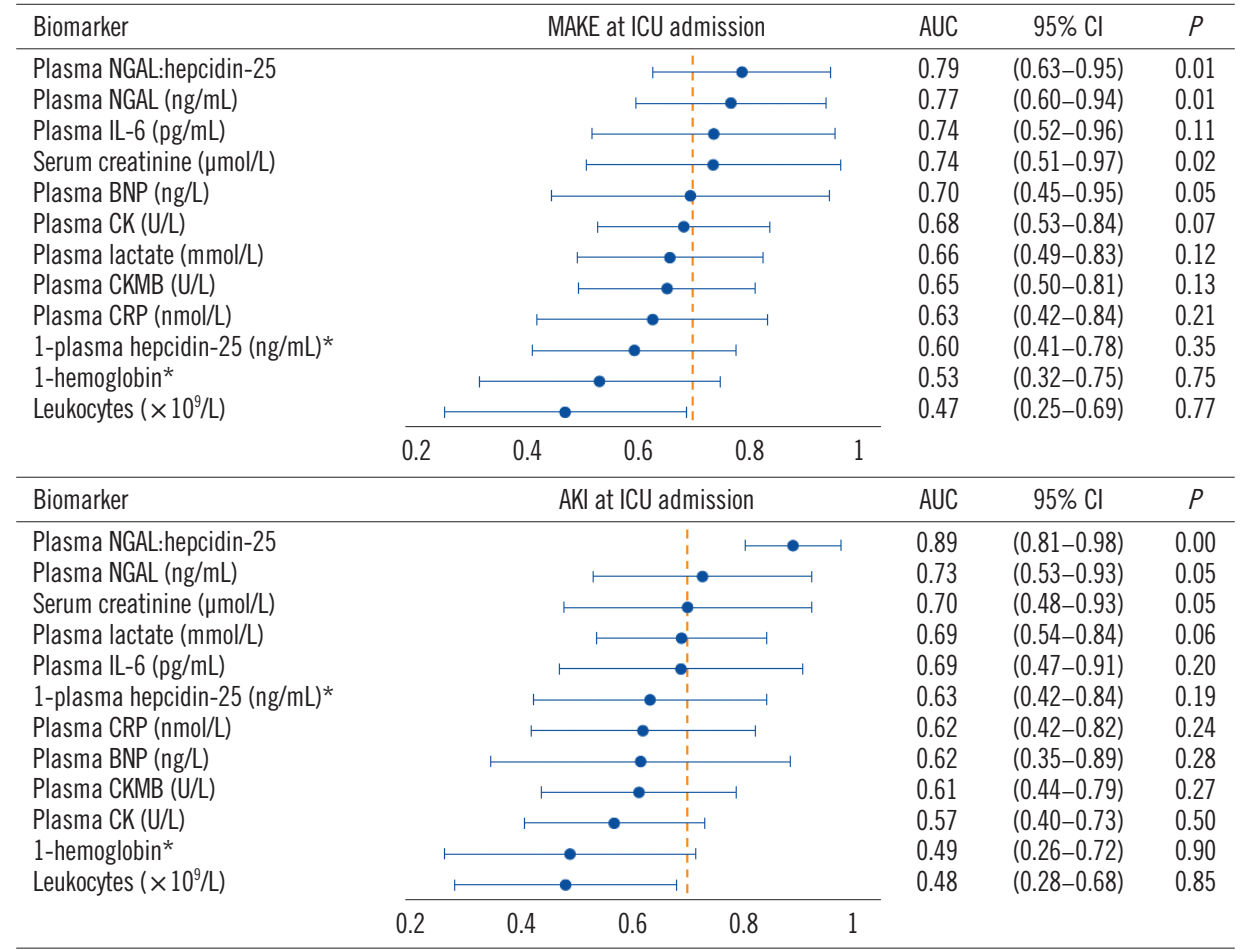

Fig. 1. AUCs of blood biomarkers at ICU admission to predict postoperative MAKE or AKI. Missing values: plasma NGAL (MAKE: $N=1 / 9$, no MAKE: $N=2 / 91 ; A K I: ~ N=2 / 9$, no AKI: $N=0 / 91$ ), hepcidin-25 (MAKE: $N=0 / 9$, no MAKE: $N=1 / 91 ; A K I: N=0 / 9$, no AKI: $N=1 / 91$ ), plasma NGAL:hepcidin-25 (MAKE: $N=1 / 9$, no MAKE: $N=2 / 91 ; A K I: N=2 / 9$, no AKI: $N=1 / 91$ ). *Indicates biomarker with inverse relationship to endpoints.

Abbreviations: AKI, acute kidney injury; AUC, area under the ROC curve; BNP, B-type natriuretic peptide; CK, creatine kinase; CKMB, creatine kinase myocardial band; CRP, C-reactive protein; ICU, intensive care unit; IL, interleukin; MAKE, major adverse kidney events; NGAL, neutrophil gelatinase-associated lipocalin. 
$A K I$ with an $A \cup C>0.7$. For both MAKE and AKI, plasma NGAL: hepcidin-25 showed the highest discriminatory value at ICU admission (MAKE: AUC $=0.79$ [95\% Cl 0.63-0.95], $P=0.007$; AKI: $A \cup C=0.89$ [0.81-0.98], $P=0.001$ ). At a cut-off of $\geq 0.9$ $(\mathrm{N}=33 / 100$ patients, $33 \%$ ), the sensitivity and specificity of the ratio were $87.5 \%$ and $70.8 \%$ for MAKE and $100 \%$ and $71.1 \%$ for AKI, respectively. None of the other biomarkers at ICU admission, including hepcidin-25, CRP, BNP, lactate, creatine kinase, creatine kinase myocardial band (CKMB), 1-hemoglobin, and leukocytes, were discriminative for the prediction of MAKE or AKI (AUC <0.7).

\section{Value of the biomarker for reclassification of patients with} or without MAKE or AKI

The addition of plasma NGAL:hepcidin-25 increased cfNRI for MAKE and AKI after adjustment for confounders (Table 2). Plasma NGAL:hepcidin-25 improved the reclassification index, cfNRI, because of correct reclassification of patients who did not develop MAKE or AKI. No biomarker improved IDI for MAKE or AKI.

\section{Covariate adjustment of biomarker predictive value for primary endpoint MAKE}

The predictive value of the best biomarker at ICU admission, plasma NGAL:hepcidin-25, was adjusted for the covariates mentioned under statistical analysis. At ICU admission, using a cut-off value of $\geq 0.9$ ( $N=33 / 100$ patients, $33 \%$ ), the ratio was an independent predictor of MAKE (adjusted OR $=16.34$ [95\% Cl 1.77-150.49], $P=0.014)$, whereas, in the same model, $\mathrm{Cr}$, was not $(P=0.590)$.

\section{AUC comparison of biomarkers for MAKE and AKI prediction}

Plasma NGAL:hepcidin-25 was compared with other biomarkers and classical kidney function parameters at ICU admission for the prediction of MAKE and AKI (Table 3). MAKE-predictive value was similar for plasma NGAL:hepcidin- 25 and $\mathrm{Cr}$, but the ratio outperformed $\mathrm{Cr}$ for $\mathrm{AKI}$ prediction (AUC $=0.89[95 \% \mathrm{Cl}$ $0.81-0.98$ ] vs. 0.63 [0.37-0.89], AUC difference $=0.26$ [95\% Cl 0.00-0.53]) (Table 3).

\section{AUC comparison of plasma biomarkers versus urinary biomarkers for MAKE and AKI prediction}

Using AUCs of urinary biomarkers at ICU admission from our previous publication [16], none of the AUC comparisons revealed an advantage of urinary biomarkers over plasma biomarkers, except that of urinary hepcidin-25, which was more

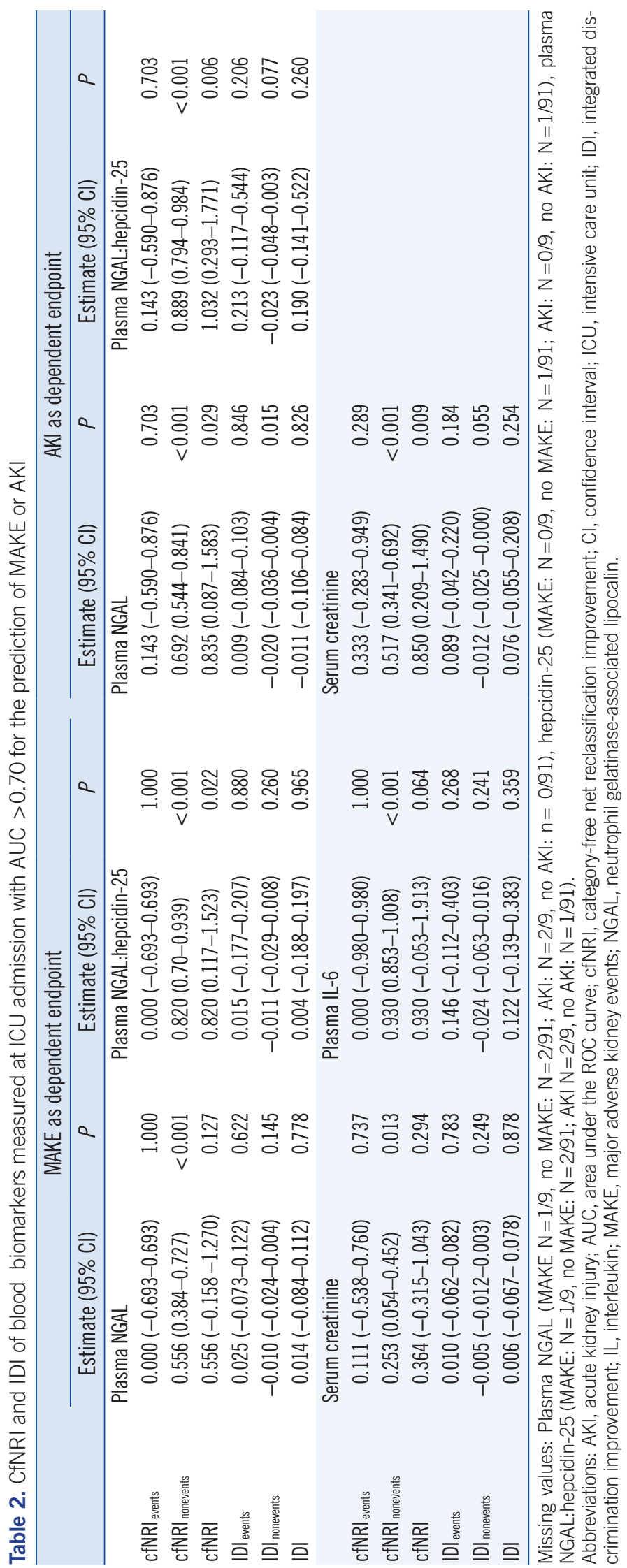


Table 3. Comparison of plasma NGAL:hepcidin-25 versus other biomarkers at ICU admission for MAKE and AKI

\begin{tabular}{|c|c|c|c|c|}
\hline \multirow{2}{*}{ Biomarkers at ICU admission (events: MAKE/AKI) } & \multicolumn{2}{|c|}{ MAKE } & \multicolumn{2}{|c|}{ AKI } \\
\hline & AUC $(95 \% \mathrm{Cl})$ & $P$ & AUC $(95 \% \mathrm{Cl})$ & $P$ \\
\hline \multirow{2}{*}{$\begin{array}{l}\text { Plasma NGAL:hepcidin- } 25 \text { vs. serum creatinine } \\
\text { ( } \mathrm{N}=8 \text { of } 97 \text { patients } / \mathrm{N}=7 \text { of } 97 \text { patients) }\end{array}$} & $0.79(0.63-0.95) ;$ & 0.599 & 0.89 (0.81-0.98); & 0.051 \\
\hline & $0.72(0.46-0.97)$ & & $0.63(0.37-0.89)$ & \\
\hline \multirow{2}{*}{$\begin{array}{l}\text { Plasma NGAL:hepcidin- } 25 \text { vs. urinary output } \\
\text { ( } N=8 \text { of } 97 \text { patients } / \mathrm{N}=7 \text { of } 97 \text { patients) }\end{array}$} & $0.79(0.63-0.95)$ & 0.894 & $0.89(0.81-0.98)$ & 0.116 \\
\hline & $0.78(0.62-0.93)$ & & $0.74(0.56-0.92)$ & \\
\hline \multirow{2}{*}{$\begin{array}{l}\text { Plasma NGAL:hepcidin- } 25 \text { vs. urinary protein } \\
\text { ( } \mathrm{N}=8 \text { of } 97 \text { patients/ } \mathrm{N}=7 \text { of } 97 \text { patients) }\end{array}$} & $0.79(0.63-0.95)$ & 0.482 & 0.89 (0.81-0.98); & 0.030 \\
\hline & $0.73(0.57-0.88)$ & & $0.66(0.45-0.88)$ & \\
\hline \multirow{2}{*}{$\begin{array}{l}\text { Plasma NGAL:hepcidin- } 25 \text { vs. plasma NGAL } \\
\text { ( } \mathrm{N}=8 \text { of } 97 \text { patients/ } \mathrm{N}=7 \text { of } 97 \text { patients) }\end{array}$} & $0.79(0.63-0.95) ;$ & 0.881 & $0.89(0.81-0.98)$ & 0.111 \\
\hline & $0.77(0.57-0.88)$ & & $0.73(0.54-0.93)$ & \\
\hline \multirow{2}{*}{$\begin{array}{l}\text { Plasma NGAL:hepcidin-25 vs. 1-plasma hepcidin-25* } \\
\text { ( } \mathrm{N}=8 \text { of } 97 \text { patients/ } \mathrm{N}=7 \text { of } 97 \text { patients) }\end{array}$} & $0.79(0.63-0.95) ;$ & 0.014 & $0.89(0.81-0.98)$ & 0.042 \\
\hline & $0.58(0.38-0.78)$ & & $0.71(0.52-0.91)$ & \\
\hline \multirow{2}{*}{$\begin{array}{l}\text { Plasma NGAL:hepcidin- } 25 \text { vs. plasma IL- } 6 \\
\text { ( } \mathrm{N}=4 \text { of } 88 \text { patients/ } \mathrm{N}=3 \text { of } 88 \text { patients) }\end{array}$} & $0.72(0.45-0.99) ;$ & 0.892 & $0.943(0.88-0.99)$ & 0.054 \\
\hline & $0.74(0.53-0.96)$ & & $0.67(0.39-0.95)$ & \\
\hline \multirow{2}{*}{$\begin{array}{l}\text { Plasma NGAL:hepcidin- } 25 \text { vs. urinary NGAL:hepcidin-25 } \\
\text { ( } \mathrm{N}=8 \text { of } 97 \text { patients } / \mathrm{N}=7 \text { of } 97 \text { patients) }\end{array}$} & $0.79(0.63-0.95) ;$ & 0.555 & $0.89(0.81-0.98)$ & 0.973 \\
\hline & $0.84(0.66-0.99)$ & & $0.89(0.80-0.98)$ & \\
\hline
\end{tabular}

*Indicates biomarker with inverse relationship to endpoints.

AUC of urinary NGAL:hepcidin-25 at ICU admission was used from a previous publication [16].

Abbreviations: AKI, acute kidney injury; AUC, area under the ROC curve; ICU, intensive care unit; IL, interleukin; MAKE, major adverse kidney events; NGAL, neutrophil gelatinase-associated lipocalin.

Table 4. Comparison of plasma biomarkers versus urinary biomarkers at ICU admission for MAKE and AKI

\begin{tabular}{|c|c|c|c|c|}
\hline \multirow{2}{*}{ Biomarkers at ICU admission (events: MAKE/AKI) } & \multicolumn{2}{|c|}{ MAKE } & \multicolumn{2}{|c|}{ AKI } \\
\hline & AUC $(95 \% \mathrm{Cl})$ & $P$ & AUC (95\% Cl) & $P$ \\
\hline $\begin{array}{l}\text { Plasma NGAL vs. urinary NGAL } \\
\text { ( } \mathrm{N}=8 \text { of } 98 \text { patients } / \mathrm{N}=7 \text { of } 98 \text { patients) }\end{array}$ & $\begin{array}{l}0.77(0.60-0.94) \\
0.73(0.54-0.93)\end{array}$ & 0.771 & $\begin{array}{l}0.73(0.53-0.93) \\
0.77(0.62-0.92)\end{array}$ & 0.729 \\
\hline $\begin{array}{l}\text { 1-plasma hepcidin-25* vs. 1-urinary hepcidin-25* } \\
\text { ( } \mathrm{N}=9 \text { of } 99 \text { patients/ } \mathrm{N}=9 \text { of } 99 \text { patients) }\end{array}$ & $\begin{array}{l}0.60(0.41-0.78) \\
0.83(0.72-0.94)\end{array}$ & 0.010 & $\begin{array}{l}0.63(0.42-0.84) \\
0.80(0.68-0.92)\end{array}$ & 0.103 \\
\hline $\begin{array}{l}\text { Plasma IL-6 vs. urinary IL- } 6 \\
\text { ( } N=4 \text { of } 89 \text { patients/N=4 of } 89 \text { patients) }\end{array}$ & $\begin{array}{l}0.74(0.51-0.96) \\
0.70(0.42-0.97)\end{array}$ & 0.712 & $\begin{array}{l}0.69(0.46-0.91) \\
0.69(0.46-0.99)\end{array}$ & $>0.99$ \\
\hline
\end{tabular}

*Indicates biomarker with inverse relationship to endpoints.

Abbreviations: AKI, acute kidney injury; AUC, area under the ROC curve; ICU, intensive care unit; IL, interleukin; MAKE, major adverse kidney events; NGAL, neutrophil gelatinase-associated lipocalin.

than that of plasma hepcidin-25 for MAKE prediction (Table 4).

\section{Correlation of plasma NGAL:hepcidin-25 with haptoglobin at ICU admission}

Plasma NGAL:hepcidin-25 at ICU admission did not correlate with absolute changes in serum haptoglobin from ICU admission to 24 hours postoperatively (Spearman-Rho $=0.191, P=0.071$ ).

\section{DISCUSSION}

We assessed the predictive value of classical kidney function parameters and clinical markers and kidney injury biomarkers for MAKE and AKI after cardiac surgery. We found that plasma NGAL, plasma NGAL:hepcidin-25, and $\mathrm{Cr}$ at ICU admission predicted MAKE and AKI with AUC $>0.7$. Classical kidney function parameters other than $\mathrm{Cr}$, such as urinary output and protein, plasma lactate, CRP, CKMB, or BNP at ICU admission did not predict MAKE or AKI. Only plasma NGAL:hepcidin-25 correctly reclassified patients according to their MAKE and AKI status. After adjustment for relevant confounders, plasma NGAL: hepcidin- 25 with a cut-off of $\geq 0.9$ was an independent predictor of MAKE, whereas $\mathrm{Cr}$ was not. This finding was supported by 
the numerically higher AUC of the ratio than that of $\mathrm{Cr}$ for AKI prediction. Plasma NGAL:hepcidin-25 did not correlate with postoperative plasma haptoglobin concentrations.

Urinary biomarkers might be more sensitive for histological damage (i.e., tubular injury), whereas plasma biomarkers might better detect changes in glomerular filtration function [25-27]. Previous studies have assessed the predictive performances of several kidney biomarkers representing direct or indirect components of tubular stress, such as NGAL or IL-6, or potential kidney-protective mechanisms, such as hepcidin-25 [28, 29]. A recent meta-analysis assessing the predictive value of plasma and urinary NGAL for AKI suggested that the predictive performance of plasma NGAL was better than that of urinary NGAL [13]. In a cohort of patients at risk of AKI, Yi, et al. [30] compared the AKI-predictive value of plasma and urinary NGAL and found higher AUC values for plasma NGAL. In the present cohort, the AUCs of plasma NGAL:hepcidin-25 and plasma NGAL appeared to be almost similar to those measured in urine for MAKE and AKI prediction. It is possible that the lack of direct detection of kidney tubular injury using plasma biomarkers may be compensated when plasma biomarkers are filtered in the setting of impaired glomerular filtration function (molecular weight $<60 \mathrm{kDa}$ ).

Consistent with previous findings for urinary and plasma hepcidin-25 [16, 31, 32], the plasma hepcidin-25 concentrations in this study were higher in patients without AKI than in those with subsequent AKI. Notably, the combination of plasma NGAL and plasma hepcidin-25 at ICU admission expressed as plasma NGAL:hepcidin-25 improved the predictive value of both markers, achieving AUC values $>0.79$ for the prediction of both endpoints. The weaker performance of plasma NGAL or plasma hepcidin-25 alone in reclassifying MAKE and AKI may reflect the limitations of the markers themselves or may be, at least partly, the effect of an "imperfect" gold standard, with changes in $\mathrm{Cr}$ being a poor reference standard.

Elevated plasma NGAL or IL-6 concentrations potentially indicate tubular injury, as such biomarkers may be reabsorbed or leaked back into the plasma [8]. Our findings may reflect the roles of both plasma NGAL and plasma hepcidin-25 in maintaining the homeostasis of labile-iron compounds during and after cardiopulmonary bypass [8, 31, 33]. During cardiac surgery, unbound labile iron-related injury maintained by inflammatory stress may be of specific pathophysiological importance for AKI [34]. Consistent with recent findings, our results suggest that lower concentrations of hepcidin-25 are independently associated with increased mortality in critically ill patients with AKI re- quiring KRT [35]. These observations highlight the importance of iron homeostasis in human ischemia-reperfusion injury and suggest a potentially modifiable risk factor during cardiac surgery [32]. However, in our cohort, haptoglobin did not correlate with plasma NGAL:hepcidin-25, suggesting potential alternative injury pathways of cell-free hemoglobin and inflammation in patients who have undergone cardiac surgery with cardiopulmonary bypass. The weak performance of routine biochemical markers, such as leukocyte counts, CRP, and creatine kinases, for predicting MAKE and AKI after cardiac surgery may be explained by a missed optimal sampling timepoint, lack of relationship with early postoperative tubular injury, or potential noninflammatory, non-cardiac lesion-related pathogenic tubular stress factors being active in cardiac surgery. Progress has been made in the development of new biomarkers to diagnose and manage AKIs that go beyond the current AKI definition/staging criteria [36]. Importantly, biomarker-based improved risk assessment after cardiac surgery may guide timely initiation of effective nephroprotective measures addressing hemodynamic and medication adjustments after cardiac surgery [37-40].

We investigated a representative, well-defined cohort of patients at increased risk of kidney injury after cardiac surgery. In this cohort, we previously described how a panel of urinary kidney biomarkers improved a preoperative kidney injury risk score [16], thus enabling direct comparison of the predictive values of simultaneously measured plasma and urinary biomarkers in the current study. However, in patient cohorts with only few MAKE or AKI events, larger sample sizes are necessary to derive reliable performance estimates, particularly for the estimation of classification errors. Thus, the sample size used in this study is a limitation precluding the generalization of the findings. In smaller studies, an apparently improved accuracy of prediction models may be skewed by model overfitting. Therefore, we used more conservative estimates resulting from cross-validation. Finally, as classical kidney function parameters, we assessed the predictive value of not only $\mathrm{Cr}$ but also urinary protein and output.

In conclusion, this pilot study identified the plasma NGAL: hepcidin-25 ratio as a strong and independent predictor of MAKE. This finding provides further support for the pathophysiologic relevance of labile iron activity in cardiac surgery-associated AKI. This ratio needs to be compared with classical kidney function parameters in larger studies including more patients with the target conditions. 
Plasma NGAL:Hepcidin-25 for kidney risk assessment

\section{ACKNOWLEDGEMENTS}

We acknowledge Mark Westerman, Ph.D. (1954-2017) of Intrinsic LifeSciences (La Jolla, CA, USA) for measuring hepcidin concentrations. This paper is dedicated to his memory. We thank Argid and Raimund Rutenberg for their excellent research assistance.

\section{AUTHOR CONTRIBUTIONS}

All authors made substantial contributions to the study conception, design, and analysis. Haase $\mathrm{M}$ and Haase-Fielitz A acquired patient data. Westphal S performed laboratory tests. Kropf $S$ and Albert $C$ performed statistical analyses. Albert $C$ and Albert A wrote the first draft of the manuscript. All authors participated in revising the paper and provided important intellectual contributions. All authors accepted their responsibility for the entire content of the manuscript and gave final approval of the submitted version and any revised versions submitted before acceptance.

\section{CONFLICTS OF INTEREST}

Albert $\mathrm{C}$ has received honoraria speaking for Siemens Healthineers. Haase $\mathrm{M}$ has received honoraria speaking for Abbott Diagnostics, Alere, Biosite Inc., and Siemens Healthineers, Albert A has received honoraria speaking for Abbott Diagnostics. All companies are involved in the development and marketing of kidney biomarkers.

\section{RESEARCH FUNDING}

This study was supported by grants from the German Heart Foundation (Deutsche Stiftung für Herzforschung) and from the Else Kröner-Fresenius-Stiftung, Germany.

\section{ORCID}

Christian Albert

Michael Haase

Annemarie Albert

Martin Ernst

Siegfried Kropf

Rinaldo Bellomo

Sabine Westphal https://orcid.org/0000-0002-6956-9962

https://orcid.org/0000-0001-8212-7416

https://orcid.org/0000-0002-5611-1506

https://orcid.org/0000-0003-3401-6935

https://orcid.org/0000-0002-5197-3481

https://orcid.org/0000-0002-1650-8939

https://orcid.org/0000-0002-5846-3345
Rüdiger C. Braun-Dullaeus

https://orcid.org/0000-0003-3888-6532

Anja Haase-Fielitz

https://orcid.org/0000-0001-6881-2249

Saban Elitok

https://orcid.org/0000-0002-1195-6871

\section{REFERENCES}

1. Thiele $\mathrm{RH}$, Isbell JM, Rosner MH. AKI associated with cardiac surgery. Clin J Am Soc Nephrol 2015;10:500-14.

2. Kellum JA, Lameire N, Aspelin P, Barsoum RS, Burdmann EA, Goldstein SL, et al. Kidney Disease: Improving Global Outcomes (KDIGO) Acute Kidney Injury Work Group. KDIGO clinical practice guideline for Acute Kidney Injury. Kidney Int Suppl 2012;2:1-138.

3. Thakar CV, Arrigain S, Worley S, Yared JP, Paganini EP. A clinical score to predict acute renal failure after cardiac surgery. J Am Soc Nephro 2005; 16:162-8.

4. McCullough PA, Shaw AD, Haase M, Bouchard J, Waikar SS, Siew ED, et al. Diagnosis of acute kidney injury using functional and injury biomarkers: workgroup statements from the tenth Acute Dialysis Quality Initiative Consensus Conference. Contrib Nephrol 2013;182:13-29.

5. Haase M, Bellomo R, Haase-Fielitz A. Novel biomarkers, oxidative stress, and the role of labile iron toxicity in cardiopulmonary bypass-associated acute kidney injury. J Am Coll Cardiol 2010;55:2024-33.

6. Leaf DE, Rajapurkar M, Lele SS, Mukhopadhyay B, Boerger EAS, Mc Causland FR, et al. Iron, hepcidin, and death in human AKI. J Am Soc Nephrol 2019;30:493-504.

7. Balla G, Jacob HS, Balla J, Rosenberg M, Nath K, Apple F, et al. Ferritin: a cytoprotective antioxidant strategem of endothelium. J Biol Chem 1992;267:18148-53.

8. Mori K, Lee HT, Rapoport D, Drexler IR, Foster K, Yang J, et al. Endocytic delivery of lipocalin-siderophore-iron complex rescues the kidney from ischemia-reperfusion injury. J Clin Invest 2005;115:610-21.

9. Gueret G, Lion F, Guriec N, Arvieux J, Dovergne A, Guennegan C, et al. Acute renal dysfunction after cardiac surgery with cardiopulmonary bypass is associated with plasmatic IL6 increase. Cytokine 2009;45:92-8.

10. Hur M, Kim H, Lee S, Cristofano F, Magrini L, Marino R, et al. Diagnostic and prognostic utilities of multimarkers approach using procalcitonin, B-type natriuretic peptide, and neutrophil gelatinase-associated lipocalin in critically ill patients with suspected sepsis. BMC Infect Dis 2014; 14:224.

11. O'Neal JB, Shaw AD, Billings FT. Acute kidney injury following cardiac surgery: current understanding and future directions. Crit Care 2016; 20:187.

12. Hajjar LA, Almeida JP, Fukushima JT, Rhodes A, Vincent J-L, Osawa EA, et al. High lactate levels are predictors of major complications after cardiac surgery. J Thorac Cardiovasc Surg 2013;146:455-60.

13. Albert C, Zapf A, Haase M, Röver C, Pickering JW, Albert A, et al. Neutrophil gelatinase-associated lipocalin measured on clinical laboratory platforms for the prediction of acute kidney injury and the associated need for dialysis therapy: A systematic review and meta-analysis. Am J Kidney Dis 2020;76:826-41.

14. von Elm E, Altman DG, Egger M, Pocock SJ, Gøtzsche PC, Vandenbroucke JP, et al. The Strengthening the Reporting of Observational Studies in Epidemiology (STROBE) statement: guidelines for reporting observational studies. Int J Surg 2014;12:1495-9.

15. Haase M, Haase-Fielitz A, Plass M, Kuppe H, Hetzer R, Hannon C, et al. Prophylactic perioperative sodium bicarbonate to prevent acute kid- 
ney injury following open heart surgery: A multicenter double-blinded randomized controlled trial. PLoS Med 2013;10:e1001426.

16. Albert C, Haase M, Albert A, Kropf S, Bellomo R, Westphal S, et al. Urinary biomarkers may complement the Cleveland score for prediction of adverse kidney events after cardiac surgery: A pilot study. Ann Lab Med 2020;40:131-41.

17. Bellomo R, Ronco C, Kellum JA, Mehta RL, Palevsky P, Acute Dialysis Quality Initiative workgroup. Acute renal failure-definition, outcome measures, animal models, fluid therapy and information technology needs: the Second International Consensus Conference of the Acute Dialysis Quality Initiative (ADQI) Group. Crit Care 2004;8:R204-12.

18. Englberger L, Suri RM, Li Z, Casey ET, Daly RC, Dearani JA, et al. Clinical accuracy of RIFLE and Acute Kidney Injury Network (AKIN) criteria for acute kidney injury in patients undergoing cardiac surgery. Crit Care 2011;15:R16.

19. Park CM, Kim JS, Moon HW, Park S, Kim H, Ji M, et al. Usefulness of plasma neutrophil gelatinase-associated lipocalin as an early marker of acute kidney injury after cardiopulmonary bypass in Korean cardiac patients: a prospective observational study. Clin Biochem 2015;48:44-9.

20. Tuladhar SM, Püntmann VO, Soni M, Punjabi PP, Bogle RG. Rapid detection of acute kidney injury by plasma and urinary neutrophil gelatinase-associated lipocalin after cardiopulmonary bypass. J Cardiovasc Pharmacol 2009;53:261-6.

21. Kerr KF, Meisner A, Thiessen-Philbrook H, Coca SG, Parikh CR. Developing risk prediction models for kidney injury and assessing incremental value for novel biomarkers. Clin J Am Soc Nephrol 2014;9:1488-96.

22. Mandrekar JN. Receiver operating characteristic curve in diagnostic test assessment. J Thorac Oncol 2010;5:1315-6.

23. Hanley JA and McNeil BJ. A method of comparing the areas under receiver operating characteristic curves derived from the same cases. Radiology 1983;148:839-43.

24. Pencina MJ, D'Agostino RB Sr, Steyerberg EW. Extensions of net reclassification improvement calculations to measure usefulness of new biomarkers. Stat Med 2011;30:11-21.

25. Vanmassenhove J, Vanholder R, Nagler E, Van Biesen W. Urinary and serum biomarkers for the diagnosis of acute kidney injury: an in-depth review of the literature. Nephrol Dial Transplant 2013;28:254-73.

26. Donadio C. Effect of glomerular filtration rate impairment on diagnostic performance of neutrophil gelatinase-associated lipocalin and B-type natriuretic peptide as markers of acute cardiac and renal failure in chronic kidney disease patients. Crit Care 2014;18:R39.

27. Kim H, Hur M, Lee S, Marino R, Magrini L, Cardelli P, et al. Proenkephalin, neutrophil gelatinase-associated lipocalin, and estimated glomerular filtration rates in patients with sepsis. Ann Lab Med 2017;37:38897.

28. Mikłaszewska M, Korohoda P, Zachwieja K, Mroczek T, Drożdż D, Sz- tefko K, et al. Serum interleukin 6 levels as an early marker of acute kidney injury on children after cardiac surgery. Adv Clin Exp Med 2013; 22:377-86.

29. Choi N, Rigatto C, Zappitelli M, Gao A, Christie S, Hiebert B, et al. Urinary hepcidin-25 is elevated in patients that avoid acute kidney injury following cardiac surgery. Can J Kidney Health Dis 2018;5:2054358117744224.

30. Yi A, Lee CH, Yun YM, Kim H, Moon HW, Hur M. Effectiveness of plasma and urine neutrophil gelatinase-associated lipocalin for predicting acute kidney injury in high-risk patients. Ann Lab Med 2021;41:60-7.

31. Prowle JR, Ostland V, Calzavacca P, Licari E, Ligabo EV, Echeverri JE, et al. Greater increase in urinary hepcidin predicts protection from acute kidney injury after cardiopulmonary bypass. Nephrol Dial Transplant 2012;27: 595-602.

32. Choi N, Whitlock R, Klassen J, Zappitelli M, Arora RC, Rigatto C, et al. Early intraoperative iron-binding proteins are associated with acute kidney injury after cardiac surgery. J Thorac Cardiovasc Surg 2019;157: 287-97.

33. van Swelm RPL, Wetzels JFM, Verweij VGM, Laarakkers CMM, Pertijs JCLM, van der Wijst J, et al. Renal handling of circulating and renalsynthesized hepcidin and its protective effects against hemoglobin-mediated kidney injury. J Am Soc Nephrol 2016;27:2720-32.

34. Swaminathan S. Iron homeostasis pathways as therapeutic targets in acute kidney injury. Nephron 2018;140:156-9.

35. Mårtensson J, Glassford NJ, Jones S, Eastwood GM, Young H, Peck L, et al. Urinary neutrophil gelatinase-associated lipocalin to hepcidin ratio as a biomarker of acute kidney injury in intensive care unit patients. Minerva Anestesiol 2015;81:1192-200.

36. Ostermann M, Bellomo R, Burdmann EA, Doi K, Endre ZH, Goldstein $\mathrm{SL}$, et al. Controversies in acute kidney injury: conclusions from a Kidney Disease: Improving Global Outcomes (KDIGO) Conference. Kidney Int 2020;98:294-309.

37. Albert C, Haase M, Albert A, Zapf A, Braun-Dullaeus RC, Haase-Fielitz A. Biomarker-guided risk assessment for acute kidney injury: time for clinical implementation? Ann Lab Med 2021;41:1-15.

38. Haase-Fielitz A, Elitok S, Schostak M, Ernst M, Isermann B, Albert C, et al. The effects of intensive versus routine treatment in patients with acute kidney injury. Dtsch Arztebl Int 2020;117:289-96.

39. Göcze I, Jauch D, Götz M, Kennedy P, Jung B, Zeman F, et al. Biomarker-guided intervention to prevent acute kidney injury after major surgery: the prospective randomized BigpAK study. Ann Surg 2018; 267:1013-20.

40. Meersch M, Schmidt C, Hoffmeier A, Van Aken H, Wempe C, Gerss J, et al. Prevention of cardiac surgery-associated AKI by implementing the KDIGO guidelines in high risk patients identified by biomarkers: the PrevAKI randomized controlled trial. Intensive Care Med 2017;43: 1551-61. 